\title{
Routing in WSN using ACO
}

\author{
Vaishali B Nikhade', R. N. Devikar ${ }^{2}$ \\ ${ }^{1}$ Pune University, Amrutvahini College of Engineering, Sangamner-422605
}

\begin{abstract}
Increasing lifetime of Wireless sensor network is a critical issues today. For overcoming the issue a good wireless sensor network is needed to be designed as energy efficient. Routing is one of the reasons for reducing the lifespan of wireless sensor network. By making energy efficient routing algorithm we can increase the lifespan of the network. In this paper we have used the combination of load balancing ACO algorithm with TARF to increase lifetime of wireless sensor network. This algorithm helps in energy and time consumption and provides security against attacks.
\end{abstract}

Keywords: Wireless sensors network, Load balancing, Ant Colony Optimization, Trusted Aware Routing Framework.

\section{Introduction}

Wireless sensor network consists of many small sensors to monitor the environmental condition by communicating with each other. As wireless sensor consist of many small sensor those are working by battery that cannot be charge or replaced if damage. The sensor network node sense and detect the disaster in environment [1]. Battery is the components which store the energy. Energy consumption is done by using two topology that is space between two nodes and equidistance hop. The function perform by the sensor node are acquisition, communication and data processing. Energy consumption is critical issue in WSN. Sensor nodes consist of operation such as transmitting and receiving data which uses large amount of energy. Using large amount of energy will affects the life of network [2]. Objective is to find how much energy is used by the network and will increase the lifetime of sensor nodes. Energy and time consumption also depend on the topology construction [3]. Sometime due to failure of node the data packet does not reach the destination [8]. Routing is the process of transferring the data packet from source to destination and the device use to transfer is called router. There are many routing algorithm which aim for energy efficiency of WSN. Routing algorithm $\mathrm{s}$ said to be when it consumed less amount of energy for transferring the data packet from source to destination. Currently used routing table are adaptive and fault free. Routing algorithm helps in finding shortest path to transfer data packet in network. There are algorithm based on swarm intelligence that is Bee algorithm and termite algorithm etc [4]. Load is also one of the degrees used for design the routing algorithm [6]. Previous algorithm was used for finding shortest path but while transferring the data packet the packet may or may not be transferred on time or may be lost all these problem in routing lead to waste the energy and time of nodes in network. At the same time security is must to be network because in WSN while transferring the data packet many attacker tries to accesses the data packets.

Wireless sensor network consists of many small sensors to monitor the environmental condition by communicating with each other. As wireless sensor consist of many small sensor those are working by battery that cannot be charge or replaced if damage. The sensor network node sense and detect the disaster in environment [1]. Battery is the components which store the energy. Energy consumption is done by using two topology that is space between two nodes and equidistance hop. The function perform by the sensor node are acquisition, communication and data processing. Energy consumption is critical issue in WSN. Sensor nodes consist of operation such as transmitting and receiving data which uses large amount of energy. Using large amount of energy will affects the life of network [2]. Objective is to find how much energy is used by the network and will increase the lifetime of sensor nodes. Energy and time consumption also depend on the topology construction [3].Sometime due to failure of node the data packet does not reach the destination [8]. Routing is the process of transferring the data packet from source to destination and the device use to transfer is called router. There are many routing algorithm which aim for energy efficiency of WSN. Routing algorithm $\mathrm{s}$ said to be when it consumed less amount of energy for transferring the data packet from source to destination. Currently used routing table are adaptive and fault free. Routing algorithm helps in finding shortest path to transfer data packet in network. There are algorithm based on swarm intelligence that is Bee algorithm and termite algorithm etc [4]. Load is also one of the degrees used for design the routing algorithm [6]. Previous algorithm was used for finding shortest path but while transferring the data packet the packet may or may not be transferred on time or may be lost all these problem in routing lead to waste the energy and time of nodes in network. At the same time security is must to be network because in WSN while transferring the data packet many attacker tries to accesses the data packets.

Ant Colony Optimization algorithm is a metahuristic algorithm. It is based on swarm intelligence. ACO is inspired from ant behavior in nature as the ant go for searching the food from nest to food source. They follow two movement forward movement and backward movement. Forward movement for searching food source and backward movement for keeping the searched food in the nest. They have a liquid call Pheromone when they start moving to the food source they go on depositing pheromone on the path. By following these pheromone deposition on the path the other ants also moves through the same path and find the food source and use same path to come back to their nest.

In ACO algorithm the ants acts a data packets and node acts as a router. The movement of ant i.e. backward and forward is call as foraging and trailing pheromone. As the ants 


\section{International Journal of Science and Research (IJSR) \\ ISSN (Online): 2319-7064}

Index Copernicus Value (2013): 6.14 | Impact Factor (2014): 5.611

perform forward movement for searching the food source from nest similarly the data packet perform the foraging pheromone for moving forward by searching under loaded node in the network. And trailing pheromone is performed when the ant encounter with an overloaded node which is the backward movement perform by ant in nature to put their food in the nest.

TARF is applied for achieving secure routing for network. TARF is used for guiding a network against attack which misdirect the routing based on identify theft for transferring routing information [9]. TARF secures the routing in network against misleading routing by providing security of neighboring nodes such Attacks are identified by their low security and transfer data by outwitting those attacker so that data would reach to the destination.

We have used TARF to provide security against forgery wormhole attacks in the network. We have provided security to all the nodes of the network. When node from other network enters our network and tries to accesses the data packet to be transferred in our network. It will misdirect the data packet from it path. To avoid such attacks we have provided security to all the nodes of the network.

In this paper, we are going to perform routing by load balancing here the routing is performed on the basis of selected node. we added security to the algorithm to make the network secured at the same time it will reduce the time and energy consumption of the sensor node in the network.

\section{Literature Survey}

Energy is main constraint taken while designing routing algorithm for transferring data packets. Yi Sun et.al [5] Proposed chain routing algorithm based on traffic prediction. This algorithm use traffic prediction model while electing the head node for judgment. This algorithm will avoid failure of node at the same time reconstruction of network topology. Asturias Diego et.al [7] Proposed range routing algorithm to balance the energy consumption of sensor network by using adjustable range transmission. the range of the power will be adjusted on the basis of position of node. Wang et.al [10] proposed a energy efficient routing protocol where the nodes are deployed randomly and sink are employed. The cluster head selection is done based on energy of nodes and the data transmission done by the node. Hamid Rafuri et.al [11] proposed effective lifetime aware routing algorithm is used for reducing the node failure. firstly the method is introduced which will determine the resource and number of nodes available. Harish Kumar et.al. [12] Proposed is used for path selection it select the path based on energy rather than length of path. Adam Mustala Zungun et al [13] proposed termite hill algorithm which is inspired from working of termite in nature termite go on depositing pheromone on the path and following that pheromone the other termite follow each other. Alexandros Giagkos et al [14] proposed a swarmed intelligence routing protocol. It provides multiple path for routing in wireless sensor network. It work as the Bee work in the nature for searching their food source.
In existing routing the node were not selected on the basis of load they had .i.e. work given to the node. The data packet used to select the node without checking the node capacity or load. When data packet where transferred from source to destination the data goes without checking the load of node after some time the node having more load will stop working as the battery power is not sufficient to complete the next task. In that case our data packet will not be moved in forward direction it will stop at that node itself. Our data packet will not be send to the destination and this will lead to consumption of more energy and time.

Existing system the data packet transmission was slow that caused congestion in network which will lead to data packet loss and also the data packet will be send on time. It may take more time and energy to transfer the data packets. In such case packet can be send to different place rather than to receiver in short they may get misdirected if the network is not secured.

Some routing algorithm were not having security against attack in that case the data packet are misguided and transferred to other node which are not the part of our network. These misdirecting of data packet from path will lead to data loss.

\section{Proposed Work}

\subsection{Working of ACO Algorithm}

ACO is used for load balancing in the network. ACO works according to the ants working in nature, firstly the WSN network is created we select the source node. Source node is selected in such a way that node should have more number of neighbor nodes. If one path is not working then another path can be selected for routing. Ants follow two movements forward and backward. In algorithm it is called as foraging and trailing pheromone.

\subsubsection{Forward movement}

Ants move in forward movement and find under loaded node and update the pheromone table of the under loaded node.

\subsubsection{Backward movement}

When ant is moving in forward direction in the network if encounter with an overloaded node. It comes back to the last under loaded node. If the node is under loaded then following formula is used for finding next node

$$
P_{i}\left(k_{s} e\right)=\frac{\left[\gamma\left(k_{v} e\right) \gamma \varepsilon\left(k_{v}, e\right)\right]^{\beta}}{\gamma\left(k_{v} e\right)\left[\beta\left(k_{v} e\right)\right]^{\beta}}
$$

$P_{i}$ is probability of ant which are on the present node $k$ is selecting neighbor node e, $\mathrm{k}$ is present node, e is neighbor node $\gamma$ is pheromone concentration, $\varepsilon$ desirable move for the ant, $\beta$ is pheromone deposition with move.

As the number of path by ants increase the cost also increase to decrease the cost a suicide timer for ant is set as the ant reach zero the ant get terminate. Timer selection depends. on the number of nodes 


\section{International Journal of Science and Research (IJSR) \\ ISSN (Online): 2319-7064}

Index Copernicus Value (2013): 6.14 | Impact Factor (2014): 5.611

\subsection{Pheromone tables}

Ants follow two type of pheromone updating i.e. foraging pheromone and trailing pheromone

\subsubsection{Foraging pheromone}

When ant is moving in forward movement they use foraging pheromone to update the pheromone table of under loaded nodes. For finding another node again $\mathrm{Fp}$ is calculated as follow:

$$
\mathrm{Fp}(\mathrm{s}+0.92)=(0.92-\alpha) \mathrm{Fp}(\mathrm{s})+\sum_{\mathrm{i}=1}^{\mathrm{k}} \Delta \mathrm{Fp}
$$

Where $\alpha$ is the rate of pheromone evaporation, before move $\mathrm{Fp}$, foraging pheromone after move Fp $(\mathrm{s}+0.92), \Delta \mathrm{Fp}$ is variation in Fp.

\subsubsection{Trailing pheromone}

Trailing pheromone is used when an ants encounter with an overloaded nodes and also to return back to the nest that is to the source. It will find a path for next under loaded node from an overloaded node

$$
\mathrm{Tp}(\mathrm{s}+0.92)=(0.92-\alpha) \mathrm{Tp}(\mathrm{s})+\sum_{\mathrm{i}=1}^{\mathrm{k}} \Delta \mathrm{Tp}
$$

Where $\alpha$ is rate evaporation, $\mathrm{Tp}$ is tracing before moving, Tp $(\mathrm{s}+0.92)$, after move, $\Delta \mathrm{Tp}$ is variation in $\mathrm{Tp}$.

Pheromone updating is used to identify the type of node searched by the ants. Ants by default follow the forward movement from the source they follow foraging pheromone and update the pheromone table. As ants encounter with overloaded nodes it comes back to previous under loaded node by trailing pheromone and update the pheromone table. After every successful transmission of data packet the timer will be check as the timer reach at zero the ant will terminate.

\section{Algorithm}

Initialization of pheromone table, ants;

Assign unique ID to all nodes

Declare threshold value

If

(ant unique ID = nodes unique ID)

\{

check for load

\}

Else

\{

Reject node

\}

If $($ load $([$ dst node $]>=$ threshold $))$

I

Max Tp (under load node)

update pt of both nodes

\}

else

\{

Min Fp (overload node)

Update pt of one node

If (under loaded node)

\author{
I \\ Free resource of node \\ l \\ Else \\ \{ \\ search for another under loaded node \\ \}
}

\section{Comparison of Existing and Proposed System}

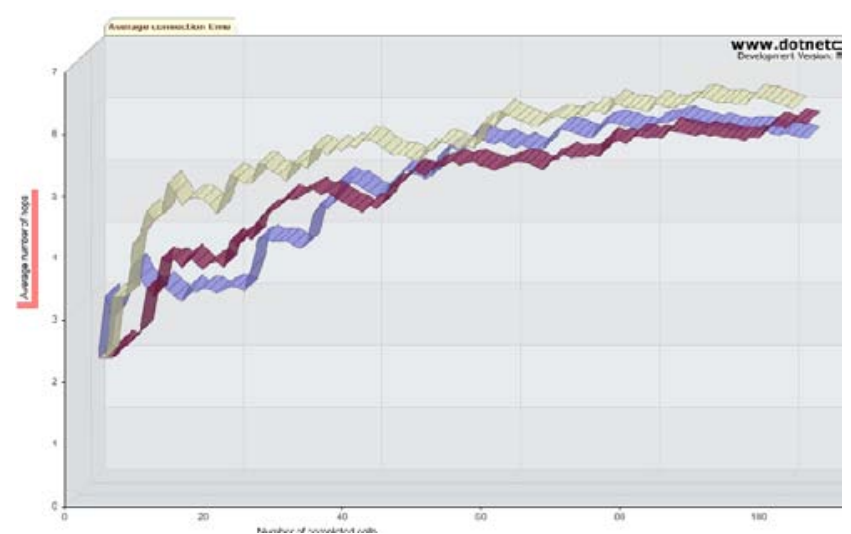

Figure 1: With ACO

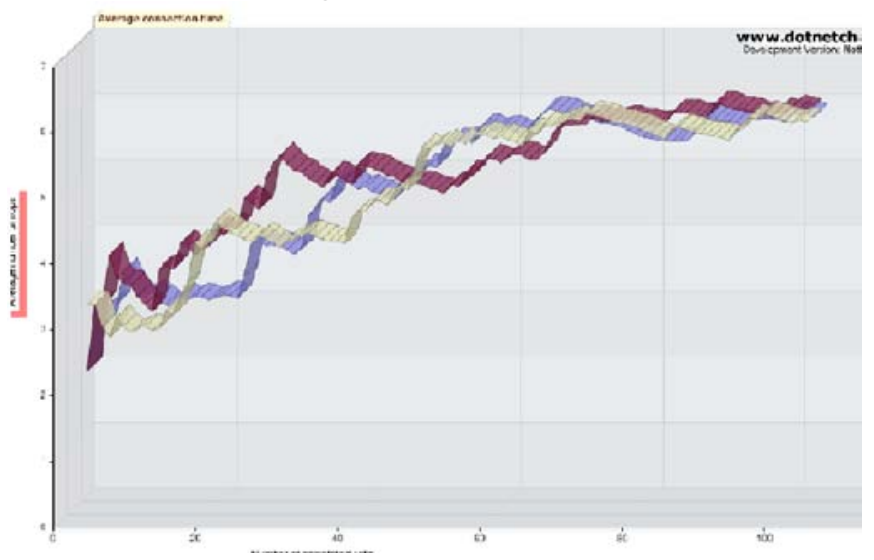

6. Conclusion

Wireless sensor network consist of both under loaded and overloaded node in the network. Overloaded node fails in data packet transmission in the network. We have used the Load balancing algorithm for checking the capacity of the node and then forwarding the data packet in the network so that the transmission will not face failure while transmission our energy and time will be consumed. We have provided security by adding TARF to the algorithm which safeguard from attacks by outside node. The above graphs prove that routing done with ACO helps reducing the energy and time consumption and this will help in prolonging the lifetime of the network.

\section{References}

[1] Jennifer Yick, Biswanath Mukherjee, Dipak Ghosal,' Wireless sensor network survey",jornal (elesvier), 2292-2330, 2008. 
[2] F.shebli,I.dayoub and J.M.Rouvaen,"Minimizing energy consumption with in wireless sensors networks", ubiquitos computing and communication journal,university of Valenciennes france.

[3] Jang, hung-Chin,Lee, Hon-Chung,Huang,JunXiand,"Optimal energy consumption for wireless sensor networks”, National Chang Chi university ,Taiwan,R.O.C

[4] D.sivakumar,B,suseela," A Survey of routing algorithms for MANET", IEEE (ICAESM-2012)

[5] Yi Sun, Lei Xu, Xin Wu, Minxuan Shen, "A Chain Routing Algorithm Based on Traffic Prediction in Wireless Sensor Networks", Communications and Network,504-507, 2013.

[6] Kumar Nishant,Pratik sharma ,Vishal Krishna, "Load Balancing of Nodes in cloud Using Ant Colony optimization",International conference on modelinng and simulation.2012.

[7] Asturias Diego Jose, Jin Wang, Imanishimwe Jean de Dieu, Sungyoung Lee "Adjustable Range Routing Algorithm based on Position for Wireless Sensor Networks" ICNIT, june2011,pp. 72-77

[8] Hong-Chi shih,Jiun-Hueiho,Bin.Yih.Liao,Jeng- shyang pan, "Fault node recovry algorithm for a wireliess sensor network”IEEE sensor journal,vol.13, no.7, july 2013.

[9] Guoxing Zhan,Weisong Shi and Julia Deng," Design and implementation of TARF : A trust aware routing framework for WSNs".IEEE 2012 Transaction on dependable and secure computing,volume 9,issue 2.

[10] Jin Wang , Zhongqi Zhang , Feng Xia , Weiwei Yuan Sungyoung Lee, "An Energy Efficient Stable ElectionBased Routing Algorithm for Wireless Sensor Networks" 2013,pp. 14301-14320;

[11] Hamid Rafiei Karkvandi, Efraim Pecht, and Orly YadidPecht,"Effective Lifetime-Aware Routing in Wireless Sensor

[12] Networks", IEEE SENSORS JOURNAL, VOL. 11, NO. 12, DECEMBER 2011

[13] . Harish Kumara, Harneet Aroraa, R.K. Singla, "EnergyAware Fisheye Routing (EA-FSR) algorithm for wireless mobile sensor networks", Egyptian Informatics Journal (2013) 14, 235-238

[14] Adamu Murtala Zungerua,n, Li-Minn Angb, Kah Phooi Sengc," Termite-hill: Performance optimized swarm

[15] Alexandros Giagkos, Myra S. Wilson," BeeIP - A Swarm Intelligence based routing for wireless ad hoc networks", Information Sciences,elesvier 2013 